\title{
Single-machine multi-agent scheduling problems with a global objective function
}

\author{
N. Huynh Tuong $\cdot$ A. Soukhal $\cdot$ J.-C. Billaut
}

(C) Springer Science+Business Media, LLC 2011

\begin{abstract}
In this paper, we consider the problem of scheduling independent jobs when several agents compete to perform their jobs on a common single processing machine. Each agent wants to minimise its cost function, which depends exclusively on its jobs and we assume that a global cost function concerning the whole set of jobs has to be minimised. This cost function may correspond to the global performance of the workshop or to the global objective of the company, independent of the objectives of the agents. Classical regular objective functions are considered and both the $\varepsilon$-constraint and a linear combination of criteria are used for finding compromise solutions. This new multi-agent scheduling problem is introduced into the literature and simple reductions with multicriteria scheduling and multi-agent scheduling problems are established. In addition, the complexity results of several problems are proposed and a dynamic programming algorithm is given.
\end{abstract}

Keywords Scheduling · Multi-agent $\cdot$ Single machine Complexity $\cdot$ Dynamic programming

\section{Introduction}

Generally in scheduling literature, the quality of a schedule is given by a measure applied to the whole set of jobs. Indeed, classical models consider all jobs to be equivalent and the quality of the global schedule is given by applying the

N. Huynh Tuong $\cdot$ A. Soukhal $\cdot$ J.-C. Billaut $(\bowtie)$ Laboratoire d'Informatique, Université François Rabelais, 64 Avenue Jean Portalis, 37200 Tours, France e-mail: jean-charles.billaut@univ-tours.fr

A. Soukhal e-mail: ameur.soukhal@univ-tours.fr same measure to all jobs without distinction. For instance, the measure may be the maximum completion time of jobs (the makespan), the total flow time of jobs or a measure related to the jobs' tardiness, e.g. maximum tardiness and the total number of tardy jobs. Introducing distinctions between jobs is generally done by the means of weights. For instance, the total weighted completion time, the total weighted tardiness or the weighted number of tardy jobs introduce such distinctions. However, in this case the same measure is still applied to all the jobs to quantify the quality of a schedule.

In a real context, these models are not always reliable. In some practical situations, it can be necessary to consider several aspects of the schedule. For instance, the mean flow time (which is equivalent to the total completion time) and the respect for due dates can be of similar importance for the decision maker. In such cases, more than one objective functions is defined and the scheduling problem enters the field of multicriteria scheduling (T'kindt and Billaut 2006 and Hoogeveen 2005 present a complete state-of-the-art survey).

In some cases, it may happen that the jobs are not equivalent and that applying the same measure to all the jobs is not useful. For instance, it is possible to consider a workshop where jobs have the following particularities: whereas some jobs may have a soft due date with allowed tardiness (which must be minimised); other jobs may have hard due dates (i.e. due dates that must be respected) and still other jobs may have no due date (e.g. production for stock). For the first type of job, the decision maker wants to minimise the maximum delay. For the second type of job he imposes that there must be no delayed jobs and for the last type of job he wants to minimise the total flow time. These jobs are assessed according to different objectives, but these jobs are in competition for the use of the machines. This problem is a multicriteria scheduling problem, where a new type 
of compromise has to be obtained. In the literature, these problems are called "interfering job sets" (Balasubramanian et al. 2009), "multi-agent scheduling" (Agnetis et al. 2000; Cheng et al. 2006) or "scheduling with competing agents" (Agnetis et al. 2004). In all these studies, the authors consider a partition of the set of jobs in competition for the use of resources, with each subset having its own objective function to optimise.

In this paper, we consider a different version of this problem in which the performance of the whole set of jobs has to be minimised. Such a problem may appear in real life situations. For instance, SKF MDGBB (Medium Deep Groove Ball Bearings) factories are workshops composed of parallel machines (see Pessan et al. 2008a, 2008b). The objective is related to the minimisation of the flow time criterion (i.e. maximising the number of items produced) and concerns the whole set of jobs, denoted by $\mathcal{N}_{0}$. Generally, the jobs that ideally, should be produced daily exceed the production capacity. To impose the production of the remaining jobs (say $\mathcal{N}_{1} \subset \mathcal{N}_{0}$ ) during the next day, another performance measure has to be applied, which is the minimisation of the number of tardy jobs (or any other due date-related measure). The measure concerning $\mathcal{N}_{0}$ is the total completion time minimisation, but the number of tardy jobs among $\mathcal{N}_{1}$ cannot exceed a given threshold. This is the field of rescheduling problems (Wu et al. 1993; Hall and Potts 2004). Another example can be found in shampoo packing systems (Mocquillon et al. 2006). Shampoo is delivered daily and stored in a dedicated storage area with a limited capacity. The problem is to find the most efficient way to pack shampoo of different types into bottles. A global objective is to maximise the production, thus reducing the setup times. At the same time, the times and quantities of future deliveries are known in advance. Thus, each type of product has to be produced daily so that its quantity never exceeds its allotted storage area. This is a typical problem where the global objective concerns all the products and where the subset of products is evaluated with another objective.

The rest of the paper is organised as follows: in Sect. 2 the problem is defined, the notations are introduced and a stateof-the-art survey is presented. The first simple reductions on existing multi-agent or multicriteria scheduling problems are also given. Section 3 deals with polynomially solvable single-machine problems. The application of simple reductions from known NP-hard problems is detailed in Sect. 4. Section 5 is devoted to the total completion time case and a pseudo-polynomial time dynamic programming is also presented.

\section{Preliminaries}

\subsection{Problem definition and notations}

A set $\mathcal{N}_{0}$ of $n$ independent jobs has to be scheduled on a single machine. We assume that all the jobs are available at time 0 , that preemption is not allowed, and that the processing times are deterministic and integers. In this paper, $p_{j}$ denotes the processing time of job $j$ and $d_{j}$ denotes its due date, where $1 \leq j \leq n$; the single machine is always available and it can process only one job at a time.

$\mathcal{N}_{k}$ denotes the $k$ th subset of jobs of $\mathcal{N}_{0}$. We assume that $\bigcup_{k=1}^{K} \mathcal{N}_{k}=\mathcal{N}_{0}$ and that $\bigcap_{k=1}^{K} \mathcal{N}_{k}=\emptyset$, with $K$ being the number of subsets. We denote by $n_{k}$ the number of jobs in $\mathcal{N}_{k}$, for $1 \leq k \leq K$.

We denote the completion time of job $j$ by $C_{j} \cdot \sum\left(w_{j}\right) C_{j}$ is the total (weighted) completion time. Additionally, $C_{\max }$ denotes the maximum completion time (makespan) and $L_{\max }$ denotes the maximum lateness, which is defined by $L_{\text {max }}=\max _{1 \leq j \leq n}\left(C_{j}-d_{j}\right)$. We denote the total (weighted) tardiness by $\sum\left(w_{j}\right) T_{j}$, where $T_{j}=\max \left(0, C_{j}-d_{j}\right)$. In addition, $U_{j}$ is equal to 1 if job $j$ is tardy, and $U_{j}$ is equal to 0 otherwise. $\sum\left(w_{j}\right) U_{j}$ denotes the (weighted) number of tardy jobs.

$f^{k}\left(\mathcal{N}_{k}\right)$ is the objective function associated with agent $k$ $(1 \leq k \leq K)$, i.e. to the subset of jobs $\mathcal{N}_{k}$ and this function is simply denoted $f^{k}$ when there is no ambiguity. $f^{0}\left(\mathcal{N}_{0}\right)$ is the objective function associated with the whole set of jobs. The problem is to find the completion times of the jobs that minimise the functions $f^{k}, \forall k \in\{0,1, \ldots, K\}$. Notice that in the case of only one agent, the problem already has two objective functions. According to the three-field notation proposed by Graham et al. (1979) and extended to multicriteria scheduling problems in T'kindt and Billaut (2006), we consider the following functions:

- $\varepsilon\left(f^{0} / f^{1}, f^{2}, \ldots, f^{K}\right)$ in the case of $K$ agents. This denotes the $\varepsilon$-constraint approach, i.e. the minimisation of $f^{0}$, subject to $f^{1} \leq \varepsilon_{1}, f^{2} \leq \varepsilon_{2}, \ldots, f^{K} \leq \varepsilon_{K}$.

- $F_{\ell}\left(f^{0}, f^{1}, f^{2}, \ldots, f^{K}\right)$ in the case of $K$ agents. This formulation indicates a linear combination of criteria, i.e. $F_{\ell}\left(f^{0}, f^{1}, f^{2}, \ldots, f^{K}\right)=\sum_{k=0}^{K} \lambda_{k} f^{k}$.

\subsection{Related literature}

The literature contains some results on multi-agent scheduling, but there are very few results when a global objective function is also considered.

Agnetis et al. (2004) consider the single machine, flow shop and open shop problems with two subsets of jobs $\mathcal{N}_{1}$ and $\mathcal{N}_{2}$. They consider the minimisation of an objective function for one subset of jobs subject to a bound for the other subset of jobs. In addition, they give some complexity 
results and dynamic programming algorithms for the singlemachine problem. The single-machine problem is also considered in Baker and Smith (2003). These authors consider several regular objective functions $\left(C_{\max }, \sum w_{j} C_{j}\right.$, $\left.L_{\max }\right)$ and they propose an algorithm for the minimisation of a linear combination of the objective functions. Complexity results are given and some polynomially solvable cases are identified. Furthermore, Yuan et al. (2005) propose some complementary results for these problems. Figure 1 summarises the results presented in Baker and Smith (2003) and Yuan et al. (2005). Cheng et al. (2006) consider a single-machine problem with $K$ disjoint subsets of jobs $\mathcal{N}_{1}, \ldots, \mathcal{N}_{K}\left(\bigcup_{i=1}^{K} \mathcal{N}_{i}=\mathcal{N}_{0}\right)$. Each job is associated with a deadline, and each subset is measured by the total number of tardy jobs. The authors prove that the decision problem denoted by $1\left|\sum w_{j} U_{j}^{1} \leq \varepsilon_{1}, \ldots, \sum w_{j} U_{j}^{K} \leq \varepsilon_{K}\right|-$ is strongly NP-hard. When the number of agents is fixed, they show that the problem can be solved in pseudo-polynomial time and they give a fully polynomial time approximation scheme. Additionally, if the weights are equal to 1 , the problem can be solved in polynomial time. Cheng et al. (2008) consider the single-machine multi-agent scheduling problem with $K$ objective functions of min-max type. The authors prove that the feasibility problem can be solved in polynomial time, even if jobs are subject to precedence constraints. Furthermore, the authors show that the problems $1\left\|\sum_{k=1}^{K}\left(L_{\max }^{k}\right), 1\right\| \sum_{k=1}^{K}\left(T_{\max }^{k}\right)$ and $1 \| \sum_{k=1}^{K}\left(\sum w_{j} C_{j}^{k}\right)$ are NP-hard and some polynomially solvable cases are identified by them. Agnetis et al. (2007) consider single-machine two-agent scheduling problems (reported in Fig. 1). Two approaches are considered: (1) the "decision problem", which is to find a solution such that all the criteria are bounded and (2) the "Pareto-optimisation problem", where the aim is to find the set of all non-dominated solutions (denoted by “\#” in Fig. 1). Some results are also given for some singlemachine multi-agent scheduling problems.

\subsection{Some simple reductions}

If the decision problem $P$ reduces to decision problem $P^{\prime}$, we use the notation $P \propto P^{\prime}$. We also use the following notations: $\Gamma^{\max }=\left\{C_{\max }, L_{\max }\right\}, \Gamma^{\Sigma}=\left\{\sum C_{j}, \sum T_{j}, \sum U_{j}\right\}$, $\Gamma^{\Sigma w}=\left\{\sum w_{j} C_{j}, \sum w_{j} T_{j}, \sum w_{j} U_{j}\right\}$ and $\Gamma=\Gamma^{\max } \cup$ $\Gamma^{\Sigma} \cup \Gamma^{\Sigma w}$. We focus on the objective functions, and $f$ stands for the problem $\alpha|\beta| f$.

Proposition 1 The following reductions hold, $\forall f^{0}$, $f^{1} \in \Gamma$ :

$1.1 f^{0} \propto F_{\ell}\left(f^{0}, f^{1}\right)$ and $f^{1}\left(\mathcal{N}_{0}\right) \propto F_{\ell}\left(f^{0}, f^{1}\left(\mathcal{N}_{1}\right)\right)$

$1.2 f^{0} \propto \varepsilon\left(f^{0} / f^{1}\right)$

$1.3 f^{1}\left(\mathcal{N}_{0}\right) \propto \varepsilon\left(f^{0} / f^{1}\left(\mathcal{N}_{1}\right)\right)$
Proof 1.1 Take the linear combination where the coefficient associated with $f^{1}$ is equal to zero; take the linear combination where the coefficient associated with $f^{0}$ is equal to zero.

1.2 Take a large value for $\varepsilon_{1}$ associated to $f^{1}$.

1.3 Finding a solution which satisfies $f^{1}\left(\mathcal{N}_{1}\right) \leq \varepsilon$ is equivalent to the decision version of problem $\alpha|\beta| f^{1}\left(\mathcal{N}_{0}\right)$ with subset $\mathcal{N}_{1}$.

Proposition 2 The following reductions from bicriteria scheduling hold, $\forall f^{0}, f^{1} \in \Gamma$ :

$2.1 F_{\ell}\left(f^{0}, f^{1}\left(\mathcal{N}_{0}\right)\right) \propto F_{\ell}\left(f^{0}, f^{1}\left(\mathcal{N}_{1}\right)\right)$

$2.2 \varepsilon\left(f^{0} / f^{1}\left(\mathcal{N}_{0}\right)\right) \propto \varepsilon\left(f^{0} / f^{1}\left(\mathcal{N}_{1}\right)\right)$

Proof Take $\mathcal{N}_{1}=\mathcal{N}_{0}$ and the problems are the same.

The consequence is that if a bicriteria scheduling problem is NP-hard, then the corresponding problem with one agent and a global objective function is NP-hard.

Proposition 3 The following reductions from multi-agent scheduling hold:

$3.1 F_{\ell}\left(f^{1}, f^{2}\left(\mathcal{N}_{2}\right)\right) \propto F_{\ell}\left(f^{1}, f^{2}\left(\mathcal{N}_{0}\right)\right), \forall f^{1} \in \Gamma, \forall f^{2} \in$ $\Gamma^{\Sigma w} \cup\left\{L_{\max }, \sum U_{j}\right\}$

$3.2 \varepsilon\left(f^{2}\left(\mathcal{N}_{2}\right) / f^{1}\right) \propto \varepsilon\left(f^{2}\left(\mathcal{N}_{0}\right) / f^{1}\right), \forall f^{1} \in \Gamma, \forall f^{2} \in$ $\Gamma^{\Sigma w} \cup\left\{L_{\max }, \sum U_{j}\right\}$

Proof If $f^{2} \in \Gamma^{\Sigma w}$, one can build an instance to problem $\alpha|\beta| F_{\ell}\left(f^{1}\left(\mathcal{N}_{1}\right), f^{2}\left(\mathcal{N}_{0}\right)\right)$ with $w_{j}=0$ for the jobs that are not in $\mathcal{N}_{1}$. Because $f^{2} \in \Gamma^{\Sigma w}, f^{2}\left(\mathcal{N}_{0}\right)=f^{2}\left(\mathcal{N}_{2}\right)$. The proof holds for the $\varepsilon$-constraint approach.

If $f_{2} \in\left\{L_{\max }, \sum U_{j}, \sum w_{j} U_{j}\right\}$, one can build an instance to problem $\alpha|\beta| F_{\ell}\left(f^{1}\left(\mathcal{N}_{1}\right), f^{2}\left(\mathcal{N}_{0}\right)\right)$ with $d_{j}=H V$ for each job $j \notin \mathcal{N}_{1}$ (HV being an high value). Because $f^{2} \in\left\{L_{\max }, \sum U_{j}, \sum w_{j} U_{j}\right\}$, the problems are the same. The same reasoning can be applied to the $\varepsilon$-constraint approach.

Proposition 4 The following reductions from multi-agent with a global objective function hold:

$4.1 F_{\ell}\left(f^{1}\left(\mathcal{N}_{1}\right), f^{0}\right) \propto F_{\ell}\left(f^{1}\left(\mathcal{N}_{0}\right), f^{0}\right), \forall f^{1} \in \Gamma^{\Sigma w} \cup$ $\left\{L_{\max }, \sum U_{j}\right\}, \forall f^{0} \in \Gamma$.

$4.2 \varepsilon\left(f^{0} / f^{1}\left(\mathcal{N}_{1}\right)\right) \propto \varepsilon\left(f^{0} / f^{1}\left(\mathcal{N}_{0}\right)\right), \forall f^{1} \in \Gamma^{\Sigma w} \cup\left\{L_{\max }\right.$, $\left.\sum U_{j}\right\}, \forall f^{0} \in \Gamma$.

Proof See the proof for Proposition 3.1.

These simple reductions are shown in Figs. 2 and 3.

Tables 1 and 2 summarise the complexity results that are established in the rest of this paper for single-machine problems (' $\mathrm{H}$ ' indicates an NP-hard problem, ' $\mathrm{P}$ ' indicates a polynomial problem and 'o' indicates an open problem). 
Fig. 1 Some complexity results on multi-agent scheduling problems

\begin{tabular}{|c|c|c|}
\hline Problem & Complexity & Reference \\
\hline $1 \| F_{l}\left(C_{\max }\left(\mathcal{N}_{1}\right), C_{\max }\left(\mathcal{N}_{2}\right)\right)$ & Polynomial & Baker and Smith (2003) \\
\hline $1 \| F_{l}\left(L_{\max }\left(\mathcal{N}_{1}\right), L_{\max }\left(\mathcal{N}_{2}\right)\right)$ & Polynomial & Baker and Smith (2003), Yuan et al. (2005) \\
\hline $1 \| F_{l}\left(\sum w_{j} C_{j}\left(\mathcal{N}_{1}\right), \sum w_{j} C_{j}\left(\mathcal{N}_{2}\right)\right)$ & Polynomial & Baker and Smith (2003) \\
\hline $1 \| F_{l}\left(C_{\max }\left(\mathcal{N}_{1}\right), L_{\max }\left(\mathcal{N}_{2}\right)\right)$ & Polynomial & Baker and Smith (2003) \\
\hline $1 \| F_{l}\left(C_{\max }\left(\mathcal{N}_{1}\right), \sum w_{j} C_{j}\left(\mathcal{N}_{2}\right)\right)$ & Polynomial & Baker and Smith (2003) \\
\hline $1 \| F_{l}\left(\sum C_{j}\left(\mathcal{N}_{1}\right), L_{\max }\left(\mathcal{N}_{2}\right)\right)$ & Polynomial & Yuan et al. (2005) \\
\hline $1 \| F_{l}\left(\sum w_{j} C_{j}\left(\mathcal{N}_{1}\right), L_{\max }\left(\mathcal{N}_{2}\right)\right)$ & Strongly NP-hard & Baker and Smith (2003) \\
\hline $1 \| F_{l}\left(C_{\max }\left(\mathcal{N}_{1}\right), L_{\max }\left(\mathcal{N}_{2}\right), \sum w_{j} C_{j}\left(\mathcal{N}_{3}\right)\right)$ & Strongly NP-hard & Baker and Smith (2003) \\
\hline $1 \| \sum_{k}\left(L_{\max }\left(\mathcal{N}_{k}\right)\right)$ & Ordinary NP-Hard & Cheng et al. (2008) \\
\hline $1 \| \sum_{k}\left(T_{\max }\left(\mathcal{N}_{k}\right)\right)$ & Ordinary NP-Hard & Cheng et al. (2008) \\
\hline $1 \| \sum_{k}\left(\max w_{j} C_{j}\left(\mathcal{N}_{k}\right)\right)$ & Strongly NP-Hard & Cheng et al. (2008) \\
\hline $1 \| \varepsilon\left(f_{\max }\left(\mathcal{N}_{1}\right) / f_{\max }\left(\mathcal{N}_{2}\right)\right)$ & Polynomial & Agnetis et al. (2004) \\
\hline $1 \| \varepsilon\left(\sum w_{j} C_{j}\left(\mathcal{N}_{1}\right) / C_{\max }\left(\mathcal{N}_{2}\right)\right)$ & Ordinary NP-Hard & Agnetis et al. (2004) \\
\hline $1 \| \varepsilon\left(\sum w_{j} C_{j}\left(\mathcal{N}_{1}\right) / L_{\max }\left(\mathcal{N}_{2}\right)\right)$ & Strongly NP-Hard & Ng et al. (2006) \\
\hline $1 \| \varepsilon\left(\sum w_{j} C_{j}\left(\mathcal{N}_{1}\right) / \sum U_{j}\left(\mathcal{N}_{2}\right)\right)$ & Strongly NP-Hard & Ng et al. (2006) \\
\hline $1 \| \varepsilon\left(\sum C_{j}\left(\mathcal{N}_{1}\right) / f_{\max }\left(\mathcal{N}_{2}\right)\right)$ & Polynomial & Agnetis et al. (2004) \\
\hline $1 \| \varepsilon\left(\sum U_{j}\left(\mathcal{N}_{1}\right) / f_{\max }\left(\mathcal{N}_{2}\right)\right)$ & Polynomial & Agnetis et al. (2004) \\
\hline $1 \| \varepsilon\left(\sum U_{j}\left(\mathcal{N}_{1}\right) / \sum U_{j}\left(\mathcal{N}_{2}\right)\right)$ & Polynomial & Agnetis et al. (2004) \\
\hline $1 \| \varepsilon\left(\sum C_{j}\left(\mathcal{N}_{1}\right) / \sum U_{j}\left(\mathcal{N}_{2}\right)\right)$ & Open* & \\
\hline $1 \| \varepsilon\left(\sum w_{j} C_{j}\left(\mathcal{N}_{1}\right) / \sum U_{j}\left(\mathcal{N}_{2}\right)\right)$ & Ordinary NP-Hard & Agnetis et al. (2004) \\
\hline $1 \| \varepsilon\left(\sum C_{j}\left(\mathcal{N}_{1}\right) / \sum C_{j}\left(\mathcal{N}_{2}\right)\right)$ & Ordinary NP-Hard & Agnetis et al. (2004) \\
\hline $1 \| \#\left(f_{\max }\left(\mathcal{N}_{1}\right), f_{\max }\left(\mathcal{N}_{2}\right)\right)$ & Polynomial & Agnetis et al. (2007) \\
\hline $1 \| \#\left(\sum w_{j} C_{j}\left(\mathcal{N}_{1}\right), f_{\max }\left(\mathcal{N}_{2}\right)\right)$ & Exponential & Agnetis et al. (2007) \\
\hline $1 \| \#\left(\sum C_{j}\left(\mathcal{N}_{1}\right), f_{\max }\left(\mathcal{N}_{2}\right)\right)$ & Polynomial & Agnetis et al. (2007) \\
\hline $1 \| \#\left(\sum U_{j}\left(\mathcal{N}_{1}\right), f_{\max }\left(\mathcal{N}_{2}\right)\right)$ & Polynomial & Agnetis et al. (2007) \\
\hline $1 \| \#\left(\sum U_{j}\left(\mathcal{N}_{1}\right), \sum U_{j}\left(\mathcal{N}_{2}\right)\right)$ & Polynomial & Agnetis et al. (2007) \\
\hline $1 \| \#\left(\sum C_{j}\left(\mathcal{N}_{1}\right), \sum U_{j}\left(\mathcal{N}_{2}\right)\right)$ & Polynomial & Agnetis et al. (2007) \\
\hline $1 \| \#\left(\sum w_{j} C_{j}(\mathcal{N} 1), \sum U_{j}\left(\mathcal{N}_{2}\right)\right)$ & Polynomial & Agnetis et al. (2007) \\
\hline $1 \| \#\left(\sum C_{j}\left(\mathcal{N}_{1}\right), \sum C_{j}\left(\mathcal{N}_{2}\right)\right)$ & Exponential & Agnetis et al. (2007) \\
\hline
\end{tabular}

Fig. 2 Simple reductions between objective functions from single objective and bicriteria scheduling

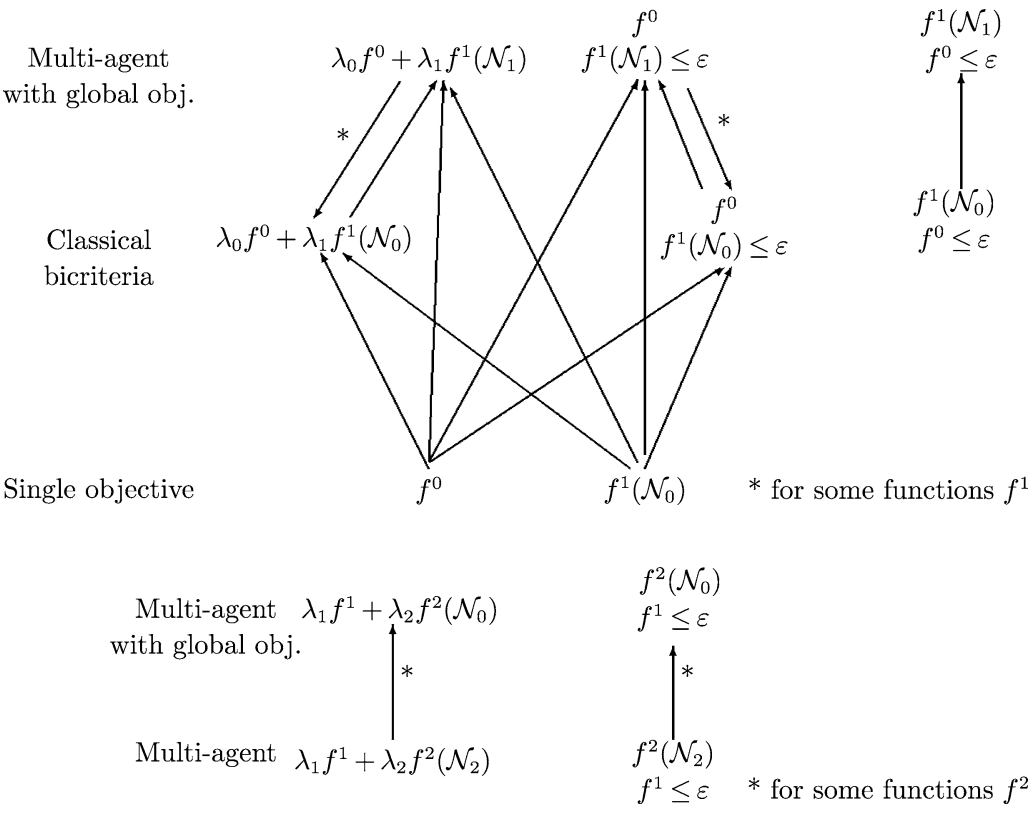

Fig. 3 Simple reductions between objective functions from multi-agent scheduling
Proposition 5 The following problems can be solved in polynomial time:

$5.11 \| F_{\ell}\left(L_{\max }^{0}, C_{\max }^{1}\right)$ and $1 \| \varepsilon\left(L_{\max }^{0} / C_{\max }^{1}\right)$

$5.21 \| F_{\ell}\left(L_{\max }^{0}, L_{\max }^{1}\right)$ and $1 \| \varepsilon\left(L_{\max }^{0} / L_{\max }^{1}\right)$

Proof 5.1 Suppose that jobs are numbered in EDD order. All the sequences $\operatorname{EDD}\left(\mathcal{N}_{1} \cup\left\{n_{1}+1, \ldots, j\right\}\right) / / E D D(\{j+$ 
Table 1 Complexity results in the case of a linear combination of criteria. Notice that this matrix is a priori not symmetric

\begin{tabular}{lllllllll}
\hline$\lambda_{1} f^{1}\left(\mathcal{N}_{1}\right)$ & $\lambda f^{0}\left(\mathcal{N}_{0}\right)$ \\
\cline { 2 - 8 } & \multicolumn{10}{l}{$C_{\max }^{0} L_{\max }^{0} \sum C_{j}^{0} \sum T_{j}^{0} \sum U_{j}^{0} \sum w_{j} C_{j}^{0} \sum w_{j} T_{j}^{0} \sum w_{j} U_{j}^{0}$} \\
\hline$C_{\max }^{1}$ & $\mathrm{P}$ & $\mathrm{P}$ & $\mathrm{P}$ & $\mathrm{H}$ & $\mathrm{o}$ & $\mathrm{P}$ & $\mathrm{H}$ & $\mathrm{H}$ \\
$L_{\max }^{1}$ & $\mathrm{P}$ & $\mathrm{P}$ & $\mathrm{P}$ & $\mathrm{H}$ & $\mathrm{o}$ & $\mathrm{H}$ & $\mathrm{H}$ & $\mathrm{H}$ \\
$\sum C_{j}^{1}$ & $\mathrm{P}$ & $\mathrm{o}$ & $\mathrm{P}$ & $\mathrm{H}$ & $\mathrm{H}$ & $\mathrm{P}$ & $\mathrm{H}$ & $\mathrm{H}$ \\
$\sum T_{j}^{1}$ & $\mathrm{H}$ & $\mathrm{H}$ & $\mathrm{H}$ & $\mathrm{H}$ & $\mathrm{H}$ & $\mathrm{H}$ & $\mathrm{H}$ & $\mathrm{H}$ \\
$\sum U_{j}^{1}$ & $\mathrm{P}$ & $\mathrm{o}$ & $\mathrm{H}$ & $\mathrm{H}$ & $\mathrm{o}$ & $\mathrm{H}$ & $\mathrm{H}$ & $\mathrm{H}$ \\
$\sum w_{j} C_{j}^{1}$ & $\mathrm{P}$ & $\mathrm{H}$ & $\mathrm{P}$ & $\mathrm{H}$ & $\mathrm{H}$ & $\mathrm{P}$ & $\mathrm{H}$ & $\mathrm{H}$ \\
$\sum w_{j} T_{j}^{1}$ & $\mathrm{H}$ & $\mathrm{H}$ & $\mathrm{H}$ & $\mathrm{H}$ & $\mathrm{H}$ & $\mathrm{H}$ & $\mathrm{H}$ & $\mathrm{H}$ \\
$\sum w_{j} U_{j}^{1}$ & $\mathrm{H}$ & $\mathrm{H}$ & $\mathrm{H}$ & $\mathrm{H}$ & $\mathrm{H}$ & $\mathrm{H}$ & $\mathrm{H}$ & $\mathrm{H}$ \\
\hline
\end{tabular}

Table 2 Complexity results after simple reductions in the case of the $\varepsilon$-constraint

\begin{tabular}{lllllllll}
\hline$f^{1}\left(\mathcal{N}_{1}\right) \leq \varepsilon_{1} \frac{\operatorname{Min} f^{0}\left(\mathcal{N}_{0}\right)}{17}$ \\
\cline { 2 - 8 }$C_{\max }^{0} L_{\max }^{0} \sum C_{j}^{0} \sum T_{j}^{0} \sum U_{j}^{0} \sum w_{j} C_{j}^{0} \sum w_{j} T_{j}^{0} \sum w_{j} U_{j}^{0}$ \\
\hline$C_{\max }^{1}$ & $\mathrm{P}$ & $\mathrm{P}$ & $\mathrm{P}$ & $\mathrm{H}$ & $\mathrm{o}$ & $\mathrm{H}$ & $\mathrm{H}$ & $\mathrm{H}$ \\
$L_{\max }^{1}$ & $\mathrm{P}$ & $\mathrm{P}$ & $\mathrm{P}$ & $\mathrm{H}$ & $\mathrm{o}$ & $\mathrm{H}$ & $\mathrm{H}$ & $\mathrm{H}$ \\
$\sum C_{j}^{1}$ & $\mathrm{P}$ & $\mathrm{o}$ & $\mathrm{H}$ & $\mathrm{H}$ & $\mathrm{H}$ & $\mathrm{H}$ & $\mathrm{H}$ & $\mathrm{H}$ \\
$\sum T_{j}^{1}$ & $\mathrm{H}$ & $\mathrm{H}$ & $\mathrm{H}$ & $\mathrm{H}$ & $\mathrm{H}$ & $\mathrm{H}$ & $\mathrm{H}$ & $\mathrm{H}$ \\
$\sum U_{j}^{1}$ & $\mathrm{P}$ & $\mathrm{o}$ & $\mathrm{H}$ & $\mathrm{H}$ & $\mathrm{o}$ & $\mathrm{H}$ & $\mathrm{H}$ & $\mathrm{H}$ \\
$\sum w_{j} C_{j}^{1}$ & $\mathrm{P}$ & $\mathrm{H}$ & $\mathrm{H}$ & $\mathrm{H}$ & $\mathrm{H}$ & $\mathrm{H}$ & $\mathrm{H}$ & $\mathrm{H}$ \\
$\sum w_{j} T_{j}^{1}$ & $\mathrm{H}$ & $\mathrm{H}$ & $\mathrm{H}$ & $\mathrm{H}$ & $\mathrm{H}$ & $\mathrm{H}$ & $\mathrm{H}$ & $\mathrm{H}$ \\
$\sum w_{j} U_{j}^{1}$ & $\mathrm{H}$ & $\mathrm{H}$ & $\mathrm{H}$ & $\mathrm{H}$ & $\mathrm{H}$ & $\mathrm{H}$ & $\mathrm{H}$ & $\mathrm{H}$ \\
\hline
\end{tabular}

$1, j+2, \ldots, n\})$ for all $j \in\left\{n_{1}+1, n_{1}+2, \ldots, n\right\}$ are tested. The best sequence with the linear combination gives an optimal solution for the function $F_{\ell}\left(L_{\max }^{0}, C_{\max }^{1}\right)$ and the best sequence that satisfies $C_{\max }\left(\mathcal{N}_{1}\right) \leq \varepsilon$ gives an optimal solution for function $L_{\max }^{0}$. The problem can be solved in $O\left(n^{2}\right)$ time. The formal proof can be obtained by pairwise interchange arguments.

5.2 There exists an optimal solution such that the jobs of $\mathcal{N}_{1}$ and the jobs of $\mathcal{N} \backslash \mathcal{N}_{1}$ are sorted in EDD order. Of course, the global sequence obtained may not follow the EDD order.

Let us consider the problem $1 \| \varepsilon\left(L_{\max }^{0} / L_{\max }^{1}\right)$. Let edd denote the global EDD sequence and let $e d d^{1}$ denote the EDD sequence for the jobs of $\mathcal{N}_{1}$. An optimal algorithm is given in Table 3. This algorithm ensures, firstly, that the epsilon-constraint is verified. Then, the jobs are scheduled according to $e d d$ order to the greatest extent possible. This algorithm finds the optimal solution in $O(n \log n)$ time.

Now let us consider the $1 \| F_{\ell}\left(L_{\max }^{0}, L_{\max }^{1}\right)$ problem. The polynomial time dynamic programming algorithm proposed by Yuan et al. (2005) in the multi-agent case can be applied to solve this problem.
Notice that problems $1 \| F_{\ell}\left(L_{\max }^{0}, \sum C_{j}^{1}\right)$ and $1 \| \varepsilon\left(L_{\max }^{0} /\right.$ $\sum C_{j}^{1}$ ) remain open.

Proposition 6 The following problems can be solved in polynomial time:

$6.11 \| F_{\ell}\left(\sum C_{j}^{0}, C_{\max }^{1}\right)$ and $1 \| \varepsilon\left(\sum C_{j}^{0} / C_{\max }^{1}\right)$

$6.21 \| F_{\ell}\left(\sum C_{j}^{0}, L_{\max }^{1}\right)$ and $1 \| \varepsilon\left(\sum C_{j}^{0} / L_{\max }^{1}\right)$

Proof 6.1 An optimal solution always exists with the jobs in $\mathcal{N}_{1}$ and the jobs in $\mathcal{N} \backslash \mathcal{N}_{1}$ being sequenced in the shortest processing time (SPT) order. Furthermore, for the makespan objective, only the completion time of the last job of $\mathcal{N}_{1}$ has to be considered. Thus, the jobs before the last job of $\mathcal{N}_{1}$ are sequenced in SPT order, whether or not they belong to $\mathcal{N}_{1}$. Let us suppose that the jobs of $\mathcal{N}_{1}$ in SPT are numbered as follows: $\left\{1,2, \ldots, n_{1}\right\}$. Let us also suppose that the jobs of $\mathcal{N} \backslash \mathcal{N}_{1}$ are $\left\{n_{1}+1, n_{1}+2, \ldots, n\right\}$. We evaluate the sequences $\operatorname{SPT}\left(\mathcal{N}_{1} \cup\left\{n_{1}+1, \ldots, j\right\}\right) / / S P T(\{j+1, j+$ $2, \ldots, n\})$ for all $j \in\left\{n_{1}+1, n_{1}+2, \ldots, n\right\}$, where $a / / b$ stands for the concatenation of $a$ and $b$. The best sequence is the optimal solution of the problem. This algorithm can be implemented in $O(n \log n)$ time.

6.2 Problem $1 \| \varepsilon\left(\sum C_{j}^{0} / C_{\max }^{1}\right)$ is polynomial.

If $P_{\mathcal{N} 1}=\sum_{J_{j} \in \mathcal{N}_{1}} p_{j}<\varepsilon$, there is no feasible solution. Otherwise, an optimal solution can be obtained by the following two-step algorithm:

1. determine the initial solution by ordering the jobs of $\mathcal{N}$ in SPT order

2. move the last jobs in $\mathcal{N}_{1}$ to the left in such a way that the new solution satisfies the $\varepsilon$-constraint.

The complexity is bounded by $O(n \log n)$.

6.2 Problem $1 \| F_{\ell}\left(\sum C_{j}^{0}, L_{\max }^{1}\right)$ has the same complexity as problem $1 \| F_{\ell}\left(L_{\max }^{0}, \sum C_{j}^{0}\right)$, which is solvable in $O\left(n^{2}\right)$ (see Hoogeveen 1992 and Proposition 4). Problem $1 \| \varepsilon\left(\sum C_{j}^{0} / L_{\max }^{1}\right)$ is equivalent to the $1\left|\tilde{d}_{j}\right| \sum C_{j}$ problem, which can be solved in polynomial time (Hoogeveen and Van de Velde 1995).

Proposition 7 The following problems can be solved in polynomial time:

$7.11 \| F_{\ell}\left(\sum w_{j} C_{j}^{0}, \sum w_{j}^{\prime} C_{j}^{1}\right)$

$7.21 \| F_{\ell}\left(\sum w_{j} C_{j}^{0}, C_{\max }^{1}\right)$

Proof 7.1 We set $w_{j}^{\prime \prime}=\lambda_{0} \times w_{j}+\lambda_{1} \times w_{j}^{\prime}$ and solve problem $1 \| \sum w_{j}^{\prime \prime} C_{j}^{0}$ (see Baker and Smith 2003). We deduce that problems $1 \| F_{\ell}\left(\sum w_{j} C_{j}^{0}, \sum C_{j}^{1}\right)$ and $1 \| F_{\ell}\left(\sum C_{j}^{0}\right.$, $\sum w_{j} C_{j}^{1}$ ) are also polynomially solvable.

7.2 Suppose that the jobs of $\mathcal{N}_{1}$ are numbered according to the weighted shortest processing time (WSPT) order. Apply the algorithm for 7.1 with $w_{j}^{\prime}=0, \forall j \neq n_{1}$ and $w_{n_{1}}^{\prime}=1$. 
Table 3 Algorithm for problem $1 \| \varepsilon\left(L_{\max }^{0} / L_{\max }^{1}\right)$

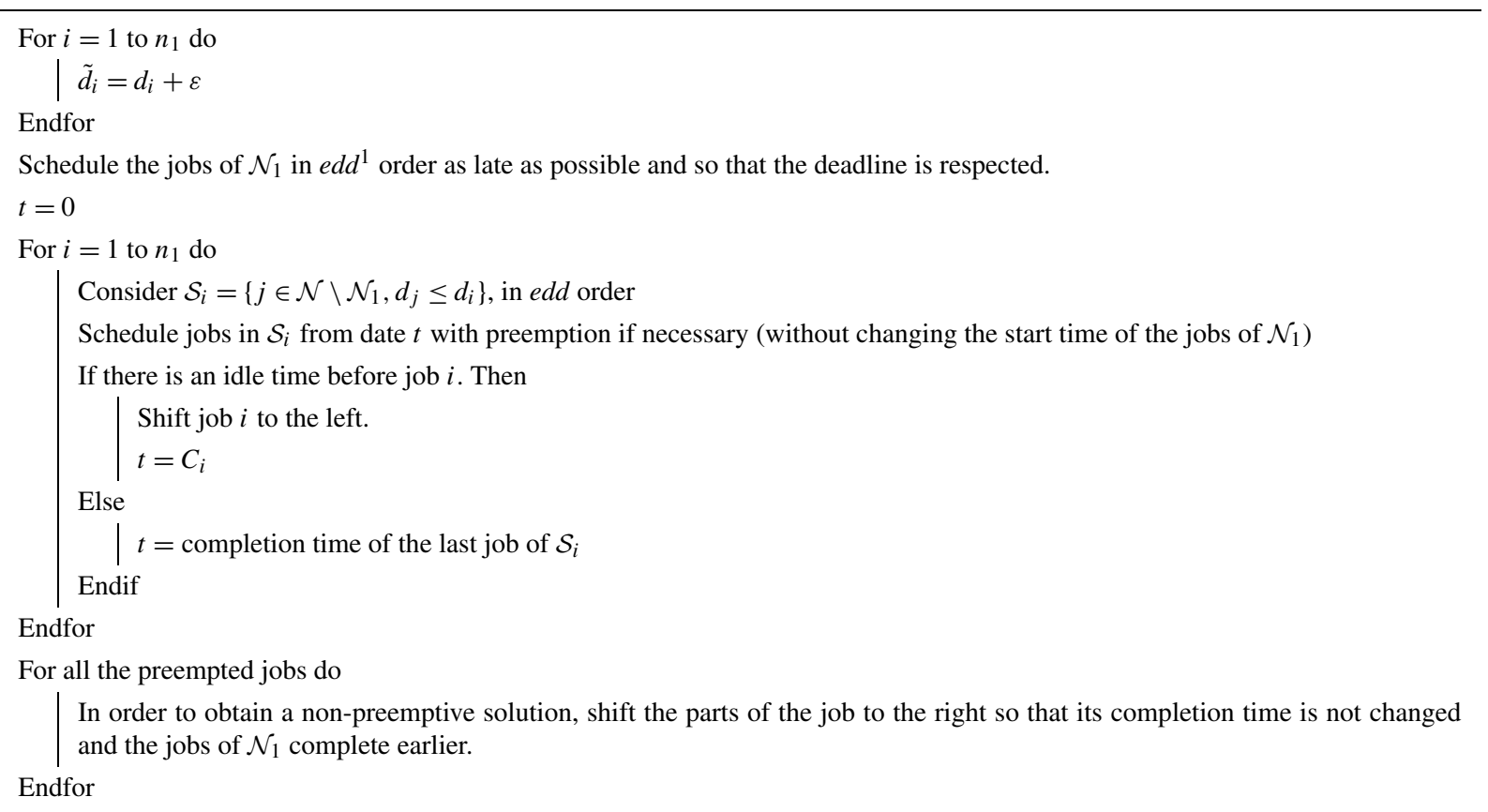

\section{Application of simple reductions from known NP-hard problems}

Proposition 8 The following problems are NP-hard:

$8.11 \| F_{\ell}\left(f^{0}, f^{1}\right) \quad$ and $1 \| \varepsilon\left(f^{0} / f^{1}\right), \quad \forall f^{0} \in\left\{\sum T_{j}\right.$, $\left.\sum w_{j} T_{j}, \sum w_{j} U_{j}\right\}, \forall f^{1} \in \Gamma$

$8.21 \| F_{\ell}\left(f^{0}, f^{1}\right)$ and $1 \| \varepsilon\left(f^{0} / f^{1}\right), \forall f^{0} \in \Gamma, \forall f^{1} \in$ $\left\{\sum T_{j}, \sum w_{j} T_{j}, \sum w_{j} U_{j}\right\}$

$8.31 \| F_{\ell}\left(L_{\max }^{0}, \sum w_{j} C_{j}^{1}\right)$ and $1 \| F_{\ell}\left(\sum w_{j} C_{j}^{0}, L_{\max }^{1}\right)$

$8.41 \| F_{\ell}\left(\sum C_{j}^{0}, \sum U_{j}^{1}\right)$ and $1 \| \varepsilon\left(\sum C_{j}^{0} / \sum U_{j}^{1}\right)$

$8.51 \| F_{\ell}\left(\sum U_{j}^{0}, \sum C_{j}^{1}\right)$ and $1 \| \varepsilon\left(\sum U_{j}^{0} / \sum C_{j}^{1}\right)$

$8.61 \| F_{\ell}\left(\sum U_{j}^{0}, \sum w_{j} C_{j}^{1}\right)$ and $1 \| \varepsilon\left(\sum U_{j}^{0} / \sum w_{j} C_{j}^{1}\right)$

Proof 8.1 Deduced from prop. 1.1 and 1.2.

8.2 Deduced from prop. 1.1 and 1.3.

8.3 This item is true, because $1 \| F_{\ell}\left(L_{\max }^{0}, \sum w_{j} C_{j}^{0}\right)$ has been proved to be NP-hard in Hoogeveen (1992) (see Proposition 2.1).

8.4 Problem $1 \| \operatorname{Lex}\left(\sum U_{j}^{0}, \sum C_{j}^{0}\right)$ is proved to be NPhard in Huo et al. (2007) (with Lex being the lexicographic minimisation, i.e., Min $\sum C_{j}^{0}$ is subject to $\sum U_{j}^{0}$ being optimal). With an appropriate choice of weights, we can show that $1\left\|\operatorname{Lex}\left(\sum U_{j}^{0}, \sum C_{j}^{0}\right) \propto 1\right\| F_{\ell}\left(\sum U_{j}^{0}, \sum C_{j}^{0}\right)$. As a consequence, this last problem is NP-hard. Thus, $1 \| F_{\ell}\left(\sum C_{j}^{0}, \sum U_{j}^{1}\right)$ is also NP-hard (see Proposition 2.1). We deduce that problem $1 \| F_{\ell}\left(\sum w_{j} C_{j}^{0}, \sum U_{j}^{1}\right)$ is also NPhard. The second property is true because $1 \| \varepsilon\left(\sum C_{j}^{0} / \sum U_{j}^{0}\right)$ has been proved NP-hard in Nelson et al. (1986) (see Proposition 2.1).
8.5 For $1 \| F_{\ell}\left(\sum U_{j}^{0}, \sum C_{j}^{1}\right)$, see the proof 8.4. 8.6 This is an immediate reduction from 8.5.

Proposition 9 The following problems are NP-hard:

$9.11 \| \varepsilon\left(L_{\max }^{0} / \sum w_{j} C_{j}^{1}\right)$

$9.21 \| \varepsilon\left(\sum w_{j} C_{j}^{0} / C_{\max }^{1}\right)$ and $1 \| \varepsilon\left(\sum w_{j} C_{j}^{0} / L_{\max }^{1}\right)$

$9.31 \| \varepsilon\left(\sum w_{j} C_{j}^{0} / \sum U_{j}^{1}\right)$

Proof 9.1 The decision version of this problem is to find a sequence so that $\sum w_{j} C_{j}^{1} \leq \varepsilon_{1}$ and $L_{\max }^{0} \leq \varepsilon_{0}$. The proposition comes because problem $1 \| \varepsilon\left(\sum w_{j} C_{j}^{1} / L_{\max }^{2}\right)$ is proved NP-hard in Agnetis et al. (2004) (see Proposition 3.2).

9.2 This proposition has the same proof as 9.1.

9.3 The proof of this proposition has the same reasoning as the proof for 9.1 because problem $1 \| \varepsilon\left(\sum w_{j} C_{j}^{1} / \sum U_{j}^{2}\right)$ is proved to be NP-hard in Agnetis et al. (2004).

\section{The case of total completion time criteria}

First we prove the NP-hardness of the $1 \| \varepsilon\left(\sum C_{j}^{0} / \sum C_{j}^{1}\right)$ problem in the case of one agent. Then, we provide a pseudo-polynomial time dynamic programming algorithm for problem $1 \| \varepsilon\left(\sum C_{j}^{0} / \sum C_{j}^{1}\right)$.

5.1 Establishing the complexity

In this section, we consider the epsilon-constraint problem for which the two objective functions are the total comple- 
Fig. 4 Initial sequence with 10 jobs

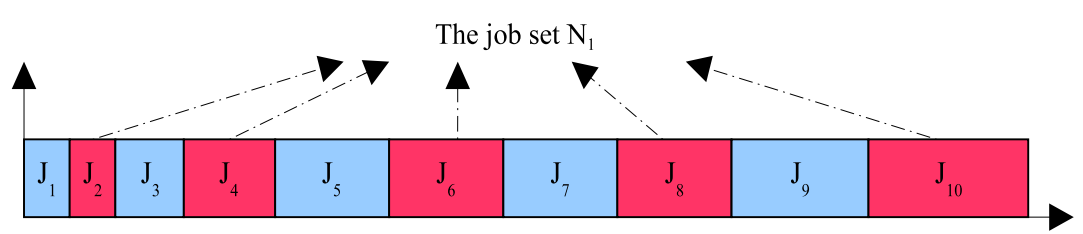

tion time, denoted by $1 \| \varepsilon\left(\sum C_{j}^{0} / \sum C_{j}^{1}\right)$. No simple reduction can be used for deriving a complexity result. We show that the problem is NP-hard.

Proposition 10 There is always an optimal solution that respects the following properties:

10.1 there is no idle time.

10.2 jobs in $\mathcal{N}_{1}$ follow the SPT order (Shortest Processing Time first).

10.3 jobs in $\left(\mathcal{N} \backslash \mathcal{N}_{1}\right)$ follow the SPT order.

10.4 if $p_{i} \leq p_{j}$, then $i$ must be scheduled before $j, \forall(i, j) \in$ $\mathcal{N}_{1} \times\left(\mathcal{N} \backslash \mathcal{N}_{1}\right)$.

Proof The first point is true because we consider regular criteria. The two next points are true because an interchange of jobs that do not follow the SPT order cannot decrease the solution's quality. The last point is true because otherwise, the permutation of $i$ and $j$ improves both $\sum C_{j}^{0}$ and $\sum C_{j}^{1}$. Note that point 4 is not true if $(i, j) \in\left(\mathcal{N} \backslash \mathcal{N}_{1}\right) \times \mathcal{N}_{1}$.

Proposition 11 Problem $1 \| \varepsilon\left(\sum C_{j}^{0} / \sum C_{j}^{1}\right)$ is NP-hard.

Proof We define the problem PWDE (PARTITION with distinct elements) below. Note that this problem has been proved NP-hard in Huynh Tuong et al. (2009):

PWDE:

Data: $\quad$ Finite set $\mathcal{B}$ of $t$ integer elements $a_{1}, a_{2}, \ldots, a_{t}$ with distinct sizes $\left(a_{i} \neq a_{j}, \forall i, j\right), \sum_{i=1}^{t} a_{i}=2 C$.

Question: Is there a subset $\mathcal{B}_{1}$ of indices such that $\sum_{i \in \mathcal{B}_{1}} a_{i}=$ $\sum_{i \in\{1,2, \ldots, t\} \backslash \mathcal{B}_{1}} a_{i}=C$ ?

We denote by $1 \mathrm{mCC}$ the decision version of problem $1 \| \varepsilon\left(\sum C_{j}^{0} / \sum C_{j}^{1}\right)$. This problem is defined by

1mCC:

Data: $\quad$ A set $\mathcal{N}_{0}$ of $n$ jobs, a subset $\mathcal{N}_{1} \subset$ $\mathcal{N}_{0}$, processing times $p_{j}$ for each job $j, 1 \leq j \leq n$, two integer values $Y_{0}$ and $Y_{1}$

Question: Is there a one-machine schedule $\sigma$ for $\mathcal{N}_{0}$ so that $\sum C_{j}^{0} \leq Y_{0}$ and $\sum C_{j}^{1} \leq Y_{1}$ ?
We must prove that PWDE $\propto 1 \mathrm{mCC}$.

We consider an arbitrary instance of PWDE and we assume without loss of generality that $a_{1}<\cdots<a_{t}$. We know that $\min _{1 \leq i \leq t-1} \frac{a_{i+1}}{a_{i}}>1$. It is always possible to find $\alpha$ and $K$ such that $1<\alpha<\min _{1 \leq i \leq t-1} \frac{a_{i+1}}{a_{i}}$ and $\alpha K \in \mathbb{N}$ (if $\frac{a_{\ell+1}}{a_{\ell}}=\min _{1 \leq i \leq t-1} \frac{a_{i+1}}{a_{i}}$, take, for instance, $\alpha=\frac{a_{\ell+1}}{a_{\ell}+1}$ and $K=a_{\ell}+1$ if $a_{\ell+1} \neq a_{\ell}+1$ or take $\alpha=\frac{10 \times a_{\ell+1}}{10 \times a_{\ell}+1}$ and $K=10 \times a_{\ell}+1$ otherwise).

Because of the definitions of $\alpha$ and $K$ we have $K a_{i}<$ $\alpha K a_{i}<K a_{i+1}<\alpha K a_{i+1}$.

Let $\beta=\alpha-1, \beta>0$ and $X=K \sum_{i=1}^{t}(2(t-i+1)+$ $(2 t-2 i+1) \alpha) \times a_{i}$.

We define an instance of problem $1 \mathrm{mCC}$ as follows: $n=$ $2 t$ and

- $p_{(2 i-1)}=K a_{i}, \forall i=1,2, \ldots, t ; p_{(2 i)}=\alpha K a_{i}, \forall i=$ $1,2, \ldots, t$;

- $Y_{1}=K(1+\alpha)\left(\sum_{i=1}^{t}(t-i+1) \times a_{i}\right)-K C ; Y_{0}=X+$ $\beta K C$;

- $\mathcal{N}_{1}=\{2,4,6, \ldots, 2 t\}$.

We define an initial solution $S^{0}=\{1,2,3, \ldots, 2 t-$ $1,2 t\}$, i.e. the sequence where the jobs are sorted according to the SPT rule (see Fig. 4).

We have

$$
\begin{aligned}
\sum_{j=1}^{n} C_{j}\left(S^{0}\right)=K a_{1}+\left(K a_{1}+\alpha K a_{1}\right)+\left(K a_{1}+\alpha K a_{1}\right. \\
\left.\quad+K a_{2}\right)+\left(K a_{1}+\alpha K a_{1}+K a_{2}+\alpha K a_{2}\right)+\cdots \\
\Rightarrow \sum_{j=1}^{n} C_{j}\left(S^{0}\right)=2 t K a_{1}+(2 t-1) \alpha K a_{1}+(2 t-2) K a_{2} \\
\quad+(2 t-3) \alpha K a_{2}+\cdots \\
\Rightarrow \sum_{j=1}^{n} C_{j}\left(S^{0}\right)=K a_{1}(2 t+(2 t-1) \alpha) \\
\quad+K a_{2}((2 t-2)+(2 t-3) \alpha)+\cdots \\
\Rightarrow \sum_{j=1}^{n} C_{j}\left(S^{0}\right)=K \sum_{i=1}^{t}(2(t-i+1) \\
\quad+(2 t-2 i+1) \alpha) \times a_{i}=X .
\end{aligned}
$$

In the same way, we obtain

$\sum_{j \in \mathcal{N}_{1}} C_{j}\left(S^{0}\right)=\left(K a_{1}+\alpha K a_{1}\right)+\left(K a_{1}+\alpha K a_{1}\right.$ 


$$
\begin{aligned}
& \left.+K a_{2}+\alpha K a_{2}\right)+\cdots \\
\Rightarrow & \sum_{j \in \mathcal{N}_{1}} C_{j}\left(S^{0}\right)=t\left(K a_{1}+\alpha K a_{1}\right)+(t-1)\left(K a_{2}\right. \\
& \left.+\alpha K a_{2}\right)+\cdots \\
\Rightarrow & \sum_{j \in \mathcal{N}_{1}} C_{j}\left(S^{0}\right)=t\left((1+\alpha) K a_{1}\right)+(t-1) \\
& \times\left((1+\alpha) K a_{2}\right)+\cdots \\
\Rightarrow & \sum_{j \in \mathcal{N}_{1}} C_{j}\left(S^{0}\right)=K(1+\alpha)\left(t a_{1}+(t-1) a_{2}+\cdots\right) \\
\Rightarrow & \sum_{j \in \mathcal{N}_{1}} C_{j}\left(S^{0}\right)=K(1+\alpha) \sum_{i=1}^{t}(t-i+1) a_{i}=Y_{1}+K C .
\end{aligned}
$$

Thus, this solution is not a feasible solution for problem 1mCC: $\sum_{j \in \mathcal{N}} C_{j}\left(S^{0}\right) \leq Y$ but $\sum_{j \in \mathcal{N}_{1}} C_{j}\left(S^{0}\right)>Y_{1}$.

- Let us suppose that the answer to PWDE is 'yes'. We are going to propose a method for permuting consecutive jobs for decreasing $\sum C_{j}^{1}$ (this method will increase $\sum C_{j}^{0}$ at the same time). We consider the set of jobs $\mathcal{G}=\left\{j \in \mathcal{N}_{0} \mid\right.$ $j=2 i$ with $\left.i \in \mathcal{B}_{1}\right\}$. Note that $\mathcal{G} \subseteq \mathcal{N}_{1}$. We define the sequence $S^{1}$ by the permutation in $S^{0}$ of each job of $\mathcal{G}$ with its predecessor: $S^{1}[j]=S^{0}[j-1], S^{1}[j-1]=S^{0}[j]$ for $j \in \mathcal{G}$ and $S^{1}[j]=S^{0}[j]$ for the other jobs.

We have to compute $\sum_{j \in \mathcal{N}_{0}} C_{j}\left(S^{1}\right)$ and $\sum_{j \in \mathcal{N}_{1}} C_{j}\left(S^{1}\right)$. We first compute these values after the permutation of only two jobs (sequence $S^{\prime}$ ).

$$
\sum_{j \in \mathcal{N}_{0}} C_{j}\left(S^{\prime}\right)=\sum_{j \in \mathcal{N}_{0}} C_{j}\left(S^{0}\right)+\left(p_{j}-p_{j-1}\right) .
$$

Thus,

$$
\begin{aligned}
& \sum_{j \in \mathcal{N}_{0}} C_{j}\left(S^{1}\right)=\sum_{j \in \mathcal{N}_{0}} C_{j}\left(S^{0}\right)+\sum_{j \in \mathcal{G}}\left(p_{j}-p_{j-1}\right) \\
& =\sum_{j \in \mathcal{N}_{0}} C_{j}\left(S^{0}\right)+\sum_{j \in \mathcal{G}}\left(\alpha K a_{j / 2}-K a_{j / 2}\right) . \\
& \Rightarrow \sum_{j \in \mathcal{N}_{0}} C_{j}\left(S^{1}\right)=\sum_{j \in \mathcal{N}_{0}} C_{j}\left(S^{0}\right)+\sum_{j \in \mathcal{G}}\left(\beta K a_{j / 2}\right) \\
& =\sum_{j \in \mathcal{N}_{0}} C_{j}\left(S^{0}\right)+\beta K \sum_{j \in \mathcal{G}} a_{j / 2} \\
& \Rightarrow \sum_{j \in \mathcal{N}_{0}} C_{j}\left(S^{1}\right) \\
& =\sum_{j \in \mathcal{N}_{0}} C_{j}\left(S^{0}\right)+\beta K \times C=X+\beta K C=Y .
\end{aligned}
$$

Similarly,

$\sum_{j \in \mathcal{N}_{1}} C_{j}\left(S^{\prime}\right)=\sum_{j \in \mathcal{N}_{1}} C_{j}\left(S^{0}\right)-p_{j-1}$

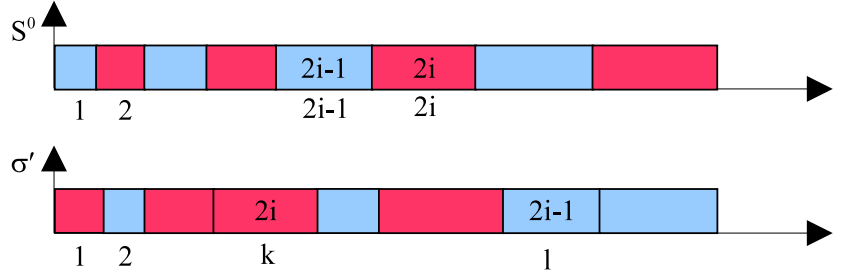

Fig. 5 Sequences $S^{0}$ and $\sigma^{\prime}$ and position of job $2 i$

Thus,

$$
\begin{aligned}
\sum_{j \in \mathcal{N}_{1}} C_{j}\left(S^{1}\right)=\sum_{j \in \mathcal{N}_{1}} C_{j}\left(S^{0}\right)-\sum_{j \in \mathcal{G}} p_{j-1} \\
\Rightarrow \sum_{j \in \mathcal{N}_{1}} C_{j}\left(S^{1}\right)=\sum_{j \in \mathcal{N}_{1}} C_{j}\left(S^{0}\right)-\sum_{j \in \mathcal{G}} K a_{j / 2} \\
\Rightarrow \sum_{j \in \mathcal{N}_{1}} C_{j}\left(S^{1}\right)=\sum_{j \in \mathcal{N}_{1}} C_{j}\left(S^{0}\right)-K C=Y_{1} \\
+K C-K C=Y_{1} .
\end{aligned}
$$

Thus, $S^{1}$ is the sequence for which the answer to $1 \mathrm{mCC}$ is 'yes'.

- Now suppose that the answer to $1 \mathrm{mCC}$ is 'yes' for sequence $\sigma$. If $\sigma$ does not respect the conditions of Proposition 10, then all the jobs are shifted to the left, the SPT rule is applied to the jobs of $\mathcal{N}_{1}$, and with respect to the jobs of $\mathcal{N}_{0} \backslash \mathcal{N}_{1}$ and each time condition 10.4 occurs, jobs $i$ and $j$ are switched. A new sequence $\sigma^{\prime}$ is obtained, so that:

- $\sum_{j \in \mathcal{N}_{0}} C_{j}\left(\sigma^{\prime}\right) \leq \sum_{j \in \mathcal{N}_{0}} C_{j}(\sigma) \leq Y$

- $\sum_{j \in \mathcal{N}_{1}} C_{j}\left(\sigma^{\prime}\right) \leq \sum_{j \in \mathcal{N}_{1}} C_{j}(\sigma) \leq Y_{1}$

- and $\sigma^{\prime}$ satisfies the conditions of Proposition 10.

We now compare $\sigma^{\prime}$ and $S^{0}$.

Let us consider the job number $2 i$. This job is in position $2 i$ in $S^{0}$ and it is in position $k$ in $\sigma^{\prime}$. Let us suppose that $k>2 i$. In this case, there is at least one job before $2 i$ in $\sigma^{\prime}$ with a bigger processing time. This job cannot belong to $\mathcal{N}_{1}$, because the jobs of $\mathcal{N}_{1}$ in $\sigma^{\prime}$ are sorted according to SPT. Thus this job belongs to $\mathcal{N} \backslash \mathcal{N}_{1}$. However, this case is not possible because of condition 10.4. Therefore, $k \leq 2 i$. Similarly, we can show that job $2 i-1$ is in position $2 i-1$ in $S^{0}$ and that it is in position $l$ in $\sigma^{\prime}$ with $l \geq 2 i-1$. This case is illustrated in Fig. 5.

We define the set of jobs $\mathcal{H}_{2 i}=\left\{j /\left(j \succ_{\sigma^{\prime}} 2 i\right) \wedge\left(p_{j}<\right.\right.$ $\left.\left.p_{2 i}\right) \wedge\left(j \in \mathcal{N}_{0} \backslash \mathcal{N}_{1}\right)\right\}$. For instance, job $2 i-1$ belongs to $\mathcal{H}_{2 i}$. These jobs are the jobs of $\mathcal{N}_{0} \backslash \mathcal{N}_{1}$ that precede job $2 i$ in $S^{0}$. 
We have $C_{2 i}\left(S^{0}\right)=C_{2 i}\left(\sigma^{\prime}\right)+\sum_{k \in \mathcal{H}_{2 i}} p_{k}$ according to the definition of $\mathcal{H}_{2 i}$.

$$
\begin{aligned}
& \Rightarrow C_{2 i}\left(\sigma^{\prime}\right)=C_{2 i}\left(S^{0}\right)-\sum_{k \in \mathcal{H}_{2 i}} p_{k} \\
& \Rightarrow \sum_{j \in \mathcal{N}_{1}} C_{j}\left(\sigma^{\prime}\right)=\sum_{j \in \mathcal{N}_{1}} C_{j}\left(S^{0}\right)-\sum_{j \in \mathcal{N}_{1}} \sum_{k \in \mathcal{H}_{j}} p_{k} \\
& \Rightarrow \sum_{j \in \mathcal{N}_{1}} C_{j}\left(\sigma^{\prime}\right)=Y_{1}+K C-\sum_{j \in \mathcal{N}_{1}} \sum_{k \in \mathcal{H}_{j}} p_{k} .
\end{aligned}
$$

Because (2) that $\sum_{j \in \mathcal{N}_{1}} C_{j}\left(\sigma^{\prime}\right) \leq Y_{1}$, we have

$$
\begin{aligned}
Y_{1}+K C & -\sum_{j \in \mathcal{N}_{1}} \sum_{k \in \mathcal{H}_{j}} p_{k} \leq Y_{1} \\
\Rightarrow K C & \leq \sum_{j \in \mathcal{N}_{1}} \sum_{k \in \mathcal{H}_{j}} p_{k} \\
\Rightarrow & K C \leq \sum_{j \in \mathcal{N}_{1}} \sum_{k \in \mathcal{H}_{j}} K a_{(k+1) / 2} \\
\Rightarrow C & \leq \sum_{j \in \mathcal{N}_{1}} \sum_{k \in \mathcal{H}_{j}} a_{(k+1) / 2 .}
\end{aligned}
$$

Due to condition 10.4, from the initial solution $S^{0}$, the position of jobs $j \in \mathcal{N}_{1}$ in $\sigma^{\prime}$ would be unchanged or moved to the left. Similarly, the position of jobs $j \in \mathcal{N}_{0} \backslash \mathcal{N}_{1}$ in $\sigma^{\prime}$ would be unchanged or moved to the right. The deviation of the completion time of a job $j \in \mathcal{N}_{0} \backslash \mathcal{N}_{1}$ between two sequences $\sigma^{\prime}$ and $S^{0}$ is determined by the total processing times of the jobs of $\mathcal{N}_{1}$ which are scheduled after $j$ in $S^{0}$, and they are also scheduled before $j$ in $\sigma^{\prime}$. For instance, in Fig. 5, the deviation of the completion time of job $2 i-1$ between two sequences $\sigma^{\prime}$ and $S^{0}$ is at least equal to $p_{2 i}$. More generally, we have

$$
\begin{aligned}
C_{2 i-1}\left(\sigma^{\prime}\right)=C_{2 i-1}\left(S^{0}\right)+\sum_{k \in \mathcal{N}_{1} \mid 2 i-1 \in \mathcal{H}_{k}} p_{k} \\
\Rightarrow \sum_{j \in \mathcal{N} \backslash \mathcal{N}_{1}} C_{j}\left(\sigma^{\prime}\right)-\sum_{j \in \mathcal{N} \backslash \mathcal{N}_{1}} C_{j}\left(S^{0}\right) \\
=\sum_{j \in \mathcal{N} \backslash \mathcal{N}_{1}} \sum_{k \in \mathcal{N}_{1} \mid j \in \mathcal{H}_{k}} p_{k} \\
\Rightarrow \sum_{j \in \mathcal{N}_{0} \backslash \mathcal{N}_{1}} C_{j}\left(\sigma^{\prime}\right)-\sum_{j \in \mathcal{N}_{0} \backslash \mathcal{N}_{1}} C_{j}\left(S^{0}\right) \\
=\sum_{k \in \mathcal{N}_{1} \mid j \in \mathcal{H}_{k}} \sum_{j \in \mathcal{N}_{0} \backslash \mathcal{N}_{1}} p_{k}=\sum_{k \in \mathcal{N}_{1}} \sum_{j \in \mathcal{H}_{k}} p_{k} .
\end{aligned}
$$

Thus, the deviation of the total completion times between two sequences $\sigma^{\prime}$ and $S^{0}$ is defined as follows:

$$
\sum_{j \in \mathcal{N}_{0}} C_{j}\left(\sigma^{\prime}\right)-\sum_{j \in \mathcal{N}_{0}} C_{j}\left(S^{0}\right)
$$

$$
\begin{aligned}
= & \left(\sum_{j \in \mathcal{N}_{1}} C_{j}\left(\sigma^{\prime}\right)-\sum_{j \in \mathcal{N}_{1}} C_{j}\left(S^{0}\right)\right) \\
& +\left(\sum_{j \in \mathcal{N}_{0} \backslash \mathcal{N}_{1}} C_{j}\left(\sigma^{\prime}\right)-\sum_{j \in \mathcal{N}_{0} \backslash \mathcal{N}_{1}} C_{j}\left(S^{0}\right)\right) .
\end{aligned}
$$

Due to (3), we have

$$
\begin{aligned}
\sum_{j \in \mathcal{N}_{1}} C_{j}\left(\sigma^{\prime}\right)-\sum_{j \in \mathcal{N}_{1}} C_{j}\left(S^{0}\right)=\sum_{j \in \mathcal{N}_{1}} \sum_{k \in \mathcal{H}_{j}} p_{k} \\
\Rightarrow \sum_{j \in \mathcal{N}_{0}} C_{j}\left(\sigma^{\prime}\right)-\sum_{j \in \mathcal{N}_{0}} C_{j}\left(S^{0}\right)=\sum_{k \in \mathcal{N}_{1}} \sum_{j \in \mathcal{H}_{k}} p_{k} \\
\quad-\sum_{j \in \mathcal{N}_{1}} \sum_{k \in \mathcal{H}_{j}} p_{k} \\
\Rightarrow \sum_{j \in \mathcal{N}_{0}} C_{j}\left(\sigma^{\prime}\right)-\sum_{j \in \mathcal{N}_{0}} C_{j}\left(S^{0}\right)=\sum_{j \in \mathcal{N}_{1}} \sum_{k \in \mathcal{H}_{j}}\left(p_{j}-p_{k}\right) .
\end{aligned}
$$

Because $p_{j}>p_{k}$ where $j \in \mathcal{N}_{1}, k \in \mathcal{H}_{j}$, we have $p_{j} \geq$ $p_{k+1}$ with $j, k+1 \in \mathcal{N}_{1}$ and $k \in \mathcal{H}_{j}$

$$
\begin{aligned}
\Rightarrow & \sum_{j \in \mathcal{N}_{0}} C_{j}\left(\sigma^{\prime}\right)-\sum_{j \in \mathcal{N}_{0}} C_{j}\left(S^{0}\right) \geq \sum_{j \in \mathcal{N}_{1}} \sum_{k \in \mathcal{H}_{j}}\left(p_{k+1}-p_{k}\right) \\
& =\sum_{j \in \mathcal{N}_{1}} \sum_{k \in \mathcal{H}_{j}}\left(\alpha K a_{(k+1) / 2}-K a_{(k+1) / 2}\right) \\
\Rightarrow & \sum_{j \in \mathcal{N}_{0}} C_{j}\left(\sigma^{\prime}\right)-\sum_{j \in \mathcal{N}_{0}} C_{j}\left(S^{0}\right) \geq \beta K \sum_{j \in \mathcal{N}_{1}} \sum_{k \in \mathcal{H}_{j}} a_{(k+1) / 2} \\
\Rightarrow & \sum_{j \in \mathcal{N}_{0}} C_{j}\left(\sigma^{\prime}\right) \geq \sum_{j \in \mathcal{N}} C_{j}\left(S^{0}\right)+\beta K \sum_{j \in \mathcal{N}_{1}} \sum_{k \in \mathcal{H}_{j}} a_{(k+1) / 2} .
\end{aligned}
$$

According to (2) and because $\sum_{j \in \mathcal{N}_{1}} \sum_{k \in \mathcal{H}_{j}} a_{(k+1) / 2} \geq C$, we have the following:

$\sum_{j \in \mathcal{N}_{0}} C_{j}\left(\sigma^{\prime}\right) \geq \sum_{j \in \mathcal{N}} C_{j}\left(S^{0}\right)+\beta K C=Y$.

Consequently, thanks to (1) and (6), we deduce that $\sum_{j \in \mathcal{N}_{0}} C_{j}\left(\sigma^{\prime}\right)=Y$.

In other words, all inequalities (4), (5) should become equalities:

$\left\{\begin{array}{l}\sum_{j \in \mathcal{N}_{1}} \sum_{k \in \mathcal{H}_{j}} a_{(k+1) / 2}=C, \\ p_{j}=p_{k+1} \quad \text { where } j \in \mathcal{N}_{1}, k \in \mathcal{H}_{j}\end{array}\right.$

Let us recall that the processing times of the jobs are all different. Hence, either $p_{j}=p_{k+1}$ (i.e. $\left|\mathcal{H}_{j}\right|=1$ ) or $\left|\mathcal{H}_{j}\right|=$ 0 where $j \in \mathcal{N}_{1}, k \in \mathcal{H}_{j}$.

$\Rightarrow\left|\mathcal{H}_{j}\right| \leq 1, \quad \forall j \in \mathcal{N}_{1}$ 
$\Rightarrow$ The equality $\sum_{j \in \mathcal{N}_{1}} \sum_{k \in \mathcal{H}_{j}} a_{(k+1) / 2}=C$

defines the subset $B_{1}$ of PWDE.

Consequently, the answer for the question of the PWDE problem is 'yes' (i.e., jobs $j$ with $\left|\mathcal{H}_{j}\right|=1$ give a subset $B_{1}$ of PWDE).

We deduce that problems $1 \| \varepsilon\left(\sum C_{j}^{0} / \sum w_{j} C_{j}^{1}\right)$, $1 \| \varepsilon\left(\sum w_{j} C_{j}^{0} / \sum C_{j}^{1}\right)$ and $1 \| \varepsilon\left(\sum w_{j} C_{j}^{0} / \sum w_{j} C_{j}^{1}\right)$ are also NP-hard.

\subsection{A dynamic programming algorithm for the $1 \| \varepsilon\left(\sum C_{j}^{0} / \sum C_{j}^{1}\right)$}

We have to both minimise $\sum C_{j}^{0}$ and respect the constraint that $\sum C_{j}^{1} \leq \varepsilon_{1}$. We assume that the jobs in $\mathcal{N}_{1}$ are numbered from 1 to $n_{1}=\left|\mathcal{N}_{1}\right|$ with $p_{1} \leq p_{2} \leq \cdots \leq p_{n_{1}}$ and that the jobs in $\mathcal{N} \backslash \mathcal{N}_{1}$ are numbered from $n_{1}+1$ to $n$ with $p_{n_{1}+1} \leq p_{n_{1}+2} \leq \cdots \leq p_{n}$. We introduce the following notations: $P$ is the sum of the processing times of all the jobs, $P_{(i, j)}=\sum_{1 \leq k \leq i} p_{k}+\sum_{n_{1}+1 \leq k \leq j} p_{k}$. We denote by $F(i, j, q)=\sum C_{j}^{0}$ the minimum cost that must arise when the jobs $\{1,2, \ldots, i\} \in \mathcal{N}_{1}$ and the jobs $\left\{n_{1}+1, n_{1}+\right.$ $2, \ldots, j\} \in \mathcal{N} \backslash \mathcal{N}_{1}$ are scheduled. $q$ corresponds to $\sum C_{j}^{1}$.

The general recursive relation is

$$
\begin{aligned}
& F\left(0, n_{1}, 0\right)=0, \\
& F(i, j, q)=+\infty, \quad \forall i>n_{1}, \forall j \leq n_{1}, \forall q, \\
& F(i, j, q)=+\infty, \quad \forall i \leq n_{1}, \forall j \in\left\{n_{1}, n_{1}+1, \ldots, n\right\}, \\
& \forall q<0 \vee q>\varepsilon_{1}, \\
& F(i, j, q)=\min \left\{\begin{array}{l}
F\left(i-1, j, q-P_{(i, j)}\right)+P_{(i, j)}, \\
F(i, j-1, q)+P_{(i, j)}
\end{array}\right\} \\
& \left(\begin{array}{l}
\forall i \in\left\{1, \ldots, n_{1}\right\} \\
\forall j \in\left\{n_{1}+1, \ldots, n\right\} \\
\forall 0 \leq q \leq \varepsilon_{1}
\end{array}\right) .
\end{aligned}
$$

The optimal solution is given by $\min _{0 \leq q \leq \varepsilon_{1}} F\left(n_{1}, n, q\right)$. The execution time of this algorithm is in $O\left(n_{1}\left(n-n_{1}\right) \varepsilon_{1}\right)$.

Proposition 12 An optimal solution to the problem $1 \| \varepsilon\left(\sum C_{j}^{0} / \sum C_{j}^{1}\right)$ can be determined in $O\left(n^{2} \varepsilon_{1}\right)$ time.

\section{Conclusion}

In this paper, we consider a new family of scheduling problems at the frontier of multi-agent and multicriteria scheduling. Subsets of jobs are in competition with the whole set of jobs for the use of resources and a compromise solution has to be found. We consider the problem of scheduling independent jobs on a single machine, without additional constraints and the objective functions are of two types: the $\varepsilon$ constraint approach and a linear combination of the criteria. We notice that these results can be extended to the case of goal programming and enumerative approaches.

Some simple reductions from multicriteria scheduling problems are established, and polynomially solvable problems and NP-hard problems are identified. A pseudopolynomial time dynamic programming algorithm is proposed for solving the case of total completion time.

This category of problems may have a lot of practical applications, and it leads to a wide area of research problems. While some complexity results remain open, some approximation schemes can be constructed for these problems as well as exact and approximated heuristic algorithms. Considering more than one agent is also a challenging perspective, as the problems combine the aspects of multicriteria scheduling and multi-agent scheduling problems, making the problems more complicated to handle. Computing the size of the Pareto set is also an interesting problem.

Acknowledgements This work has been supported by the project ANR-08-BLAN-0331-01, funded by the "National Research Agency" (ANR).

\section{References}

Agnetis, A., Mirchandani, P. B., Pacciarelli, D., \& Pacifici, A. (2000). Nondominated schedules for a job-shop with two competing users. Computational and Mathematical Organization Theory, 6, 191-217.

Agnetis, A., Mirchandani, P. B., Pacciarelli, D., \& Pacifici, A. (2004). Scheduling problems with two competing agents. Operations Research, 52, 229-242.

Agnetis, A., Pacciarelli, D., \& Pacifici, A. (2007). Multi-agent single machine scheduling. Annals of Operations Research, 150, 3-15.

Baker, K., \& Smith, J. C. (2003). A multiple-criterion model for machine scheduling. Journal of Scheduling, 6, 7-16.

Balasubramanian, H., Fowler, J., Keha, A., \& Pfund, M. (2009). Scheduling interfering job sets on parallel machines. European Journal of Operational Research, 199(1), 55-67.

Cheng, T. C. E., Ng, C. T., \& Yuan, J. J. (2006). Multi-agent scheduling on a single machine to minimize total weighted number of tardy jobs. Theoretical Computer Science, 362, 273-281.

Cheng, T. C. E., Ng, C. T., \& Yuan, J. J. (2008). Multi-agent scheduling on a single machine with max-form criteria. European Journal of Operational Research, 188, 603-609.

Garey, M. R., \& Johnson, D. S. (1979). Computers and intractability: a guide to the theory of NP-completeness. San Francisco: W.H. Freeman.

Graham, R. L., Lawler, E. L., Lenstra, J. K., \& Rinnooy Kan, A. H. G. (1979). Optimization and approximation in deterministic sequencing and scheduling: a survey. Annals of Discrete Mathematics 5, 287-326.

Hall, N. G., \& Potts, C. N. (2004). Rescheduling for new orders. Operations Research, 52(3), 440-453 +496.

Hoogeveen, H. (1992). Single machine bicriteria scheduling. $\mathrm{PhD}$ Thesis, Amsterdam. 
Hoogeveen, H. (2005). Multicriteria scheduling. European Journal of Operational Research, 167, 592-623.

Hoogeveen, H., \& Van de Velde, S. (1995). Minimizing completion time and maximum cost simultaneously is solvable in polynomial time. Operations Research Letters, 17, 205-208.

Huo, Y., Leung, J. Y.-T., \& Zhao, H. (2007). Complexity of two dual criteria scheduling problems. Operations Research Letters, 35, 211-220.

Huynh Tuong, N., Soukhal, A., \& Billaut, J.-C. (2009). Complexity of partition problem with distinct elements (Research report num. 295), University of Tours, France, March.

Mocquillon, C., Lenté, C., \& T'Kindt, V. (2006). Solution of a multicriteria shampoo production problem. In IEEE international conference on service systems and service management (IEEESSSM'06), Troyes (France) (pp. 907-911).

Nelson, R. T., Sarin, R. K., \& Daniels, R. L. (1986). Scheduling with multiple performance measures: the one-machine case. Management Science, 32(4), 464-479.
Ng, C. T., Cheng, T. C. E., \& Yuan, J. J. (2006). A note on the complexity of the problem of two-agent scheduling on a single machine. Journal of Combinatorial Optimization, 12, 387-394.

Pessan, C., Bouquard, J.-L., \& Neron, E. (2008a). An unrelated parallel machines model for an industrial production resetting problem. European Journal of Industrial Engineering, 2(2), 153-171.

Pessan, C., Bouquard, J.-L., \& Neron, E. (2008b). Genetic branch-andbound or exact genetic algorithm. In Lecture notes in computer science (Vol. 4926, pp. 136-147). Berlin: Springer.

T'kindt, V., \& Billaut, J.-C. (2006). Multicriteria scheduling: theory, models and algorithms (2nd ed.). Berlin: Springer.

Wu, S. D., Storer, R. H., \& Chang, P.-C. (1993). One-machine rescheduling heuristics with efficiency and stability as criteria. Computers \& Operations Research, 20(1), 1-14.

Yuan, J. J., Shang, W. P., \& Feng, Q. (2005). A note on the scheduling with two families of jobs. Journal of Scheduling, 8, 537-542. 